Hardy-Ramanujan Journal

Vol. 31 (2008)14-27

\title{
Arithmetical investigations of particular Wynn power series
}

\author{
Peter Bundschuh
}

\section{Introduction and results}

The power series we study here are of the following type

$$
\sum_{n=1}^{\infty} \frac{z^{n}}{\prod_{\lambda=0}^{\ell-1} R_{a(n+\lambda)+b}},
$$

where $R_{m}$ is given by

$$
R_{m}:=g u^{m}+h v^{m} .
$$

Here, and in the whole paper, $u, v, g, h$ are non-zero complex numbers with $|u|>|v|$ and such that $R_{m} \neq 0$ holds for any $m \in \mathbb{N}:=\{1,2, \ldots\} ;$ moreover, we always assume $a, \ell \in \mathbb{N}$ and $b \in \mathbb{N}_{0}:=\mathbb{N} \cup\{0\}$. Slightly more general power series than those of type (1) were first considered by Wynn [19] from the analytical point of view. He obtained, essentially, the explicit formula for the associated Padé approximants, and later others, e.g., Driver and Lubinsky [7], continued these analytical studies.

It is very natural that, for arithmetical investigations of series (1), we have to make suitable arithmetical assumptions on the parameters $u, v, g, h$ appearing in (2), and also on the point $z$. Taking, for example, $u=\frac{1}{2}(1+\sqrt{5}), v=\frac{1}{2}(1-\sqrt{5}), g=-h=\frac{1}{\sqrt{5}}$, according to Binet's formula, $R_{m}$ becomes the $m$ th Fibonacci number $F_{m}$ determined recursively by $F_{0}:=0, F_{1}:=1$ and $F_{m}:=F_{m-1}+F_{m-2}$ for $m=2,3, \ldots$ Indeed, it was the series $\sum_{n=1}^{\infty} z^{n} / F_{n}$ (in fact, a slightly more general one) which was first investigated with regard to irrationality at the only non-zero integer points $z= \pm 1$ within its disk of convergence $|z|<u$ by André-Jeannin [1]. He used a method reminiscent of the one of Apéry for his proof of the irrationality of $\zeta(3)$. A few years later, K. Väänänen and the present author [5] obtained, inter alia, a quantitative version of André-Jeannin's irrationality result on $\sum_{n=1}^{\infty} z^{n} / F_{n}, z \in$ $\{1,-1\}$, using a quantitative generalization of Nesterenko's method for linear independence. Next, Prévost [15] extended these qualitative and quantitative irrationality results to any non-zero rational point $z$ in the domain of meromorphy of the function originally defined by the power series only in its disk of convergence. In fact, his approach to analyze the Padé approximants to the series $\sum_{n=1}^{\infty} z^{n} / R_{n}$, where the sequence $\left(R_{n}\right)_{n \in \mathbb{N}}$ satisfies a second order recurrence relation, led to similar results in the case, where the rational field is replaced by any imaginary quadratic number field. More recently, Matala-aho and Prévost [12],[13] 
extended these quantitative investigations using Padé approximations for particular Heine $q$-series as constructed in closed form by Matala-aho [11].

The main aim of the present paper is to prove a rather general result (Theorem 1) on arithmetic properties of certain series, where the entering parameters are algebraic over $\mathbb{Q}$ but not necessarily of degree at most two. From this result we deduce an arithmetic consequence (Theorem 2) on power series of type (1) in the case $\ell=1$, and on their meromorphic continuation to the whole complex plane, where the sequence $\left(R_{m}\right)$ need not consist of positive rational integers nor do we use explicitly the fact that it satisfies a second order linear recurrence. Thus, we extend results of Tachiya [18] using again Borwein's simple analytic method from [3]. This method is effective in the sense that it could lead to quantitative results (compare, e.g., [4]). Nevertheless, for the sake of technical simplicity of the presentation, we restrict ourselves in the whole paper to qualitative statements only.

Theorem 1. Let $q \in \overline{\mathbb{Q}}$ be an integer whose conjugates satisfy $\left|q^{\{\sigma\}}\right|<1$ for any $\sigma \in$ $\operatorname{Aut}(\overline{\mathbb{Q}} \mid \mathbb{Q}) \backslash\{\mathrm{id}\}$, and put $K:=\mathbb{Q}(q)$. Suppose, that, for $\alpha \in K^{\times} \backslash\left\{q^{-1}, q^{-2}, \ldots\right\}$, there exists $t \in \mathbb{N}_{0}$ with $\left(\operatorname{den}\left(\alpha q^{t}\right)\right)^{2 \operatorname{deg}(q)}<|q|$. Let $\beta \in K$ be a unit satisfying $|\beta| \leq 1$ and $\left|\beta^{\{\sigma\}}\right| \geq 1$ for any $\sigma \in \operatorname{Aut}(\overline{\mathbb{Q}} \mid \mathbb{Q}) \backslash\{\mathrm{id}\}$. Then

$$
\sum_{k=1}^{\infty} \frac{\beta^{k}}{1-\alpha q^{k}} \notin K .
$$

Remark. Notice that the above condition on the denominator of $\alpha q^{t}$ implies already $|q|>1$. Note also $\operatorname{deg}(q):=[K: \mathbb{Q}]$.

From Theorem 1 we can obtain the following result, which is most appropriate for our applications.

Theorem 2. Let $K$ be an algebraic number field and $O_{K}$ its ring of integers. Assume that $u, v \in K^{\times}$have the following properties: $\frac{u}{v}$ is a unit in $O_{K}$, for any $m \in \mathbb{N}$ the inclusion $K \subset$ $\mathbb{Q}\left(\left(\frac{u}{v}\right)^{m}\right)$ holds, the inequalities $\left|\frac{u}{v}\right|>1$ and $\left|\left(\frac{u}{v}\right)^{\{\sigma\}}\right|<1$ hold for any $\sigma \in \operatorname{Aut}(\overline{\mathbb{Q}} \mid \mathbb{Q}) \backslash\{\mathrm{id}\}$. Finally, suppose $g, h \in K^{\times}$such that $\frac{g}{h}$ is a unit in $O_{K}$, and $(a, b) \in \mathbb{N} \times \mathbb{N}_{0}$. Then, for the function $\mathcal{R}(z ; a, b)$ defined in $|z|<|u|^{a}$ by the power series

$$
\sum_{n=1}^{\infty} \frac{z^{n}}{R_{a n+b}},
$$

the following holds

$$
\mathcal{R}(\gamma, a, b) \notin K
$$

if $\gamma \in K^{\times} \backslash u^{a}\left(\frac{u}{v}\right)^{a \mathbb{N}_{0}}$ satisfies the condition $\operatorname{den}\left(\gamma^{-1} v^{a}\left(\frac{u}{v}\right)^{a t}\right)^{2[K: \mathbb{Q}]}<\left|\frac{u}{v}\right|^{a}$ for some $t \in \mathbb{N}_{0}$.

Remark. Of course, the condition $K \subset \mathbb{Q}\left(\left(\frac{u}{v}\right)^{m}\right)$ means nothing but $K=\mathbb{Q}\left(\left(\frac{u}{v}\right)^{m}\right)$ for any $m \in \mathbb{N}$. 
Next, we present a slight generalization of Theorem 2 .

Theorem 3. Assume all hypotheses of Theorem 2 on $K ; u, v, g, h ; a, b$ and suppose, moreover, $\ell \in \mathbb{N}$. Then, for the meromorphic function $\mathcal{R}_{\ell}(z ; a, b)$ defined in $|z|<|u|^{a \ell}$ by the power series (1), the following alternative holds.

(i) If $\gamma \in K^{\times} \backslash u^{a \ell}\left(\frac{u}{v}\right)^{a \mathbb{N}_{0}}, \gamma \notin\left\{u^{a(\ell-\lambda)} v^{a \lambda} \mid \lambda=1, \ldots, \ell-1\right\}$ and if

$$
\operatorname{den}\left(\frac{u^{a(\ell-1)} v^{a}}{\gamma}\left(\frac{u}{v}\right)^{a t}\right)^{2[K: \mathbb{Q}]}<\left|\frac{u}{v}\right|^{a}
$$

for suitable $t \in \mathbb{N}_{0}$, then $\mathcal{R}_{\ell}(\gamma ; a, b) \notin K$.

(ii) If $\gamma \in\left\{u^{a(\ell-\lambda)} v^{a \lambda} \mid \lambda=1, \ldots, \ell-1\right\}$, then $\mathcal{R}_{\ell}(\gamma ; a, b)$ is an element of $K$, which can be explicitly determined.

Remark. For $\ell=1$, Theorem 3 reduces exactly to Theorem 2 ( $\mathcal{R}_{1}$ being, of course, our earlier $\mathcal{R}$ ) since then the case (ii) cannot occur and, moreover, in the case (i) the condition $\gamma \notin\{\ldots\}$ is void.

Our first application of Theorem 3 is the following.

Corollary 1. Let u be an algebraic integer, whose minimal polynomial (over $\mathbb{Z}$ ) has value \pm 1 at the origin, and which satisfies the condition $|u|>1>\left|u^{\{\sigma\}}\right|$ for any $\sigma \in \operatorname{Aut}(\overline{\mathbb{Q}} \mid \mathbb{Q}) \backslash\{\mathrm{id}\}$. Suppose $g, h \in K:=\mathbb{Q}(u), g h \neq 0$ such that $\frac{g}{h}$ is a unit in $O_{K}$. Then, for the meromorphic function $\mathcal{R}_{\ell}(z ; a, b)$ defined in $|z|<|u|^{a \ell}$ by the power series (1) with $R_{m}=g u^{m}+h$ (i.e., $v=1$ in (2)) the following alternative holds.

(i) If $\gamma \in K^{\times} \backslash u^{a \mathbb{N}}$ and if $\left.\operatorname{den}\left(\gamma^{-1} u^{a t}\right)\right)^{2 \operatorname{deg}(u)}<|u|^{a}$ for suitable $t \in \mathbb{N}_{0}$ with $t \geq \ell-1$, then $\mathcal{R}_{\ell}(\gamma ; a, b) \notin K$.

(ii) If $\gamma \in\left\{u^{a \lambda} \mid \lambda=1, \ldots, \ell-1\right\}$, then $\mathcal{R}_{\ell}(\gamma ; a, b)$ is an element of $K$, which may be entirely specified.

Examples of algebraic integers $u$ as appearing in Corollary 1 can be found, e.g., among the so-called Pisot numbers or Pisot-Vijayaraghavan numbers ${ }^{1}$ (PV-numbers). By definition, these are real algebraic integers $u>1$, whose conjugates $u^{\{\sigma\}}(\sigma \neq$ id if $\operatorname{deg}(u)>1)$ have all absolute values less than 1 . Hence the PV-numbers of degree 1 are exactly the rational integers $2,3,4, \ldots$. Notice that every monic non-constant polynomial

$$
g(X)=X^{d}+\sum_{\delta=0}^{d-1} a_{\delta} X^{\delta} \in \mathbb{Z}[X] \quad \text { with } 1+\sum_{\delta=0}^{d-1} a_{\delta}<0 \text { and }^{2} \quad 1+\sum_{\delta=0}^{d-2}\left|a_{\delta}\right|<\left|a_{d-1}\right|
$$

has a PV-number $u$ among its zeros, by Rouché's theorem. If $\varphi_{u} \in \mathbb{Z}[X]$ is the minimal polynomial of this $u$, then $g(x)=\varphi_{u}(X) f(X)$ holds, where $f \in \mathbb{Z}[X]$ has a representation $f(X)=\prod_{j}\left(X-u_{j}\right)$ with certain algebraic integers $u_{j}$ from the open unit disk. Note that $f(X)=1$ happens if and only if the product here is empty. Thus, $\prod_{j}\left(-u_{j}\right)=f(0)$ is a rational integer with absolute value less than 1 if the product is not empty, whence $f(0)=0$, hence $a_{0}=0$ in (6). From this consideration we conclude the following. If we choose, for

\footnotetext{
${ }^{1}$ Excellent references to this topic are the monographs [2] and [17].

${ }^{2}$ As usual, empty sums or products have to be interpreted as 0 or 1 , respectively.
} 
arbitrarily given $d \in \mathbb{N} \backslash\{1\}$, a monic polynomial $g \in \mathbb{Z}[X]$ satisfying not only the two conditions in (6) on its coefficients but also $g(0)=a_{0} \in\{1,-1\}$, then, for each degree $\geq 2$, we can find a lot of algebraic integers $u$ of this degree, which satisfy all conditions of Corollary 1 on $u$.

To mention explicitly a few PV-numbers of degree $2,3,4$, having all properties required in Corollary 1 for $u$, we make the following brief side-remark. As a matter of fact, the set $S$ of all $\mathrm{PV}$-numbers is closed on the real line. Hence it has a least element, and this is the real root $\vartheta_{0}=1.324717 \ldots$ of the polynomial $X^{3}-X-1$, an isolated point of $S$. The set $S \backslash\left\{\vartheta_{0}\right\}$ again has a least element, namely the positive real root $\vartheta_{1}=1.380277 \ldots$ of $X^{4}-X^{3}-1$. In fact, all (infinitely many) points of $S$ less than the 'golden number' $\frac{1}{2}(1+\sqrt{5})=1.618034 \ldots \in S$, sometimes denoted by $\vartheta_{\infty}$, are isolated, and this $\vartheta_{\infty}$ is the smallest limit point of $S$.

Expressions $R_{m}$ of type (2) arise, in particular, when dealing with certain binary linear recurrences, which we now discuss briefly. Let $R_{0}, R_{1}, A, B \in \mathbb{Z}, A B \neq 0$ and

$$
R_{m}=A R_{m-1}+B R_{m-2} \quad(m=2,3, \ldots) .
$$

Then all $R_{m} \in \mathbb{Z}$ and we suppose furthermore $R_{m} \neq 0$ for any $m \in \mathbb{N}$. Assume the companion polynomial $X^{2}-A X-B$ of $(7)$ to be irreducible in $\mathbb{Q}[X]$. Its roots $u, v$ are $\frac{1}{2}(A+\sqrt{\Delta})$ and $\frac{1}{2}(A-\sqrt{\Delta})$ with $\Delta:=A^{2}+4 B$ not a square. In addition, we assume $\Delta>0$. Thus, and by $A \neq 0$, we obtain $|u| \neq|v|$ and, w.l.o.g., we may suppose $|u|>|v|$ as we did from the beginning. With $g:=\left(R_{1}-R_{0} v\right) /(u-v), h:=\left(R_{0} u-R_{1}\right) /(u-v)$, each $R_{m}(m \geq 0)$ has the shape (2).

Corollary 2. Suppose that $\left(R_{m}\right) \in \mathbb{Z}^{\mathbb{N}_{0}}$ is a binary linear recurrence satisfying (7) and all subsequent assumptions. Suppose further that $\frac{u}{v}$ and $\frac{g}{h}$ are units in $O_{K}$, where $K:=\mathbb{Q}(\sqrt{\Delta})$. Then, for the meromorphic function $\mathcal{R}_{\ell}(z ; a, b)$, defined in $|z|<|u|^{a \ell}$ by the power series (1), the following alternative holds.

(i) If $\gamma \in K^{\times} \backslash\left\{u^{a \ell}\left(\frac{u}{v}\right)^{a \lambda} \mid \lambda \in \mathbb{Z}, \lambda>-\ell\right\}$ and $\operatorname{den}\left(\gamma^{-1} u^{a \ell}\left(\frac{u}{v}\right)^{a t}\right)<\left|\frac{u}{v}\right|^{a / 4}$ for suitable $t \in \mathbb{Z}, t \geq-1$, then $\mathcal{R}_{\ell}(\gamma ; a, b) \notin K$.

(ii) If $\gamma \in\left\{u^{a(\ell-\lambda)} v^{a \lambda} \mid \lambda=1, \ldots, \ell-1\right\}$, then $\mathcal{R}_{\ell}(\gamma ; a, b)$ is in $K$ and can be explicitly specified.

Remark. Note that the quotient $\frac{u}{v}$ is a unit in $O_{K}$ if $B \in\{1,-1\}$ holds in (7). Note also that the particular cases $\ell=1$ and $\ell=2$ of our last corollary imply Corollary 2 and 3 , respectively, of Tachiya [18].

Probably the most 'prominent' binary linear recurrences are the Fibonacci and Lucas sequences, which both satisfy ( 7$)$ with $A=B=1$, where one has to take $(0,1)$ and $(2,1)$, respectively, as initial pair $\left(R_{0}, R_{1}\right)$. Denoting these, as usual, by $\left(F_{m}\right)$ and $\left(L_{m}\right)$, respectively, the particular case $\ell=1$ of Corollary 2 has the following application.

Corollary 3. For the meromorphic functions $\mathcal{F}(z ; a, b)$ and $\mathcal{L}(z ; a, b)$ defined $i n^{3}|z|<\vartheta_{\infty}^{a}$ by the power series

$$
\sum_{n=1}^{\infty} \frac{z^{n}}{F_{a n+b}} \text { and } \sum_{n=1}^{\infty} \frac{z^{n}}{L_{a n+b}},
$$

\footnotetext{
${ }^{3}$ Remember the above convention $\vartheta_{\infty}:=\frac{1}{2}(1+\sqrt{5})$.
} 
respectively, one has

$$
\mathcal{F}(\gamma ; a, b), \mathcal{L}(\gamma ; a, b) \notin \mathbb{Q}(\sqrt{5})
$$

if $\gamma \in \mathbb{Q}(\sqrt{5})^{\times}$is different from the poles $(-1)^{a \lambda} \vartheta_{\infty}^{a(2 \lambda+1)}, \lambda \in \mathbb{N}_{0}$, of both functions and if $\operatorname{den}\left(\gamma^{-1} \vartheta_{\infty}^{a(2 t-1)}\right)<\vartheta_{\infty}^{a / 2}$ for suitable $t \in \mathbb{N}_{0}$. In particular, (8) holds for all $\gamma=\vartheta_{\infty}^{c}$ with $c \in \mathbb{Z} \backslash\{a, 3 a, 5 a, \ldots\}$ if $a$ is even, and with $c \in \mathbb{Z} \backslash\{a, 5 a, 9 a, \ldots\}$ if $a$ is odd; (8) holds for all $\gamma=-\vartheta_{\infty}^{c}$ with $c \in \mathbb{Z}$ if $a$ is even, and with $c \in \mathbb{Z} \backslash\{3 a, 7 a, 11 a, \ldots\}$ if $a$ is odd.

Remark. Note that the two cases $\gamma= \pm 1$ include the main result from [1] in the Fibonacci and Lucas case.

Finally, our last result is basically an application of Corollary 2 even if we will need some additional considerations for its full proof.

Corollary 4. The series

$$
\sum_{n=1}^{\infty} \frac{1}{F_{n} \cdot \ldots \cdot F_{n+\ell-1}}
$$

is not contained in the field $\mathbb{Q}(\sqrt{5})$ if $4 \nmid \ell$, but for $4 \mid \ell$ it belongs to $\mathbb{Q}(\sqrt{5}) \backslash \mathbb{Q}$. The series

$$
\sum_{n=1}^{\infty} \frac{(-1)^{n}}{F_{n} \cdot \ldots \cdot F_{n+\ell-1}}
$$

is not contained in $\mathbb{Q}(\sqrt{5})$ if $2 \nmid \ell$ or $4 \mid \ell$, but belongs to $\mathbb{Q}(\sqrt{5}) \backslash \mathbb{Q}$ if $2 \mid \ell, 4 \nmid \ell$. Exactly the same alternatives hold for the Lucas instead of the Fibonacci numbers. In particular, all series mentioned here are irrational.

Remark 1. Note that, as a consequence of our method of proof, the values of the series can be explicitly determined, at least in principle, in all cases, where they belong to $\mathbb{Q}(\sqrt{5}) \backslash \mathbb{Q}$.

Remark 2. Carlitz [6] produced reduction formulas for Fibonacci sums of type

$$
C_{\ell}:=\sum_{n=1}^{\infty} \frac{(-1)^{n[\ell / 2]}}{F_{n} \cdot \ldots \cdot F_{n+\ell-1}} \quad(\ell \in \mathbb{N})
$$

but gave no arithmetical applications. Since, modifying our earlier notation, we have $C_{\ell}=$ $\mathcal{F}_{\ell}\left((-1)^{[\ell / 2]} ; 1,0\right)$ for any $\ell \in \mathbb{N}$, our Corollary 4 implies $C_{\ell} \notin \mathbb{Q}(\sqrt{5})$ if $\ell$ is odd, and $C_{\ell} \in \mathbb{Q}(\sqrt{5}) \backslash \mathbb{Q}$ if $\ell$ is even.

We conclude this introduction by a few questions on transcendence and algebraic independence. For example, one could adopt the principle that every series in Corollary 4, whose value is not in $\mathbb{Q}(\sqrt{5})$, should be transcendental. Another good open problem, proposed by Ribenboim [16, p.60], is the algebraic independence of the three series (9) for $\ell=1,2,3$. Having in mind that Duverney, the Nishiokas and Shiokawa [8] proved the transcendence of series like

$$
\sum_{n=1}^{\infty} F_{n}^{-\ell}, \sum_{n=1}^{\infty}(-1)^{n} F_{n}^{-\ell}, \sum_{n=1}^{\infty} L_{n}^{-\ell}, \sum_{n=1}^{\infty}(-1)^{n} L_{n}^{-\ell}
$$


for any even $\ell \in \mathbb{N}$, and Elsner, Shimomura and Shiokawa [9] very recently the algebraic independence of the three numbers

$$
\sum_{n=1}^{\infty} F_{n}^{-2}, \sum_{n=1}^{\infty} F_{n}^{-4}, \sum_{n=1}^{\infty} F_{n}^{-6}
$$

(and the same if replacing the $F$ 's by the $L$ 's, or considering the alternating cases), questions as above are probably not entirely hopeless.

\section{Proof of Theorem 1}

Denoting the sum in (3) by $\omega$, we obtain with $\alpha_{t}:=\alpha q^{t}$

$$
\beta^{-t}\left(\omega-\sum_{k=1}^{t} \frac{\beta^{k}}{1-\alpha q^{k}}\right)=\sum_{k=1}^{\infty} \frac{\beta^{k}}{1-\alpha_{t} q^{k}}=: \omega_{t}
$$

for every $t \in \mathbb{N}_{0}$. For the statement (3) it is enough to prove $\omega_{t} \notin K$, where, by a suitable choice of $t$, we may suppose $\left(\operatorname{den}\left(\alpha_{t}\right)\right)^{2 \operatorname{deg}(q)}<|q|$ as well as $\left|\alpha_{t}\right|>1$ and $\left|\alpha_{t}^{\{\sigma\}}\right| \leq 1$ for any $\sigma \in \operatorname{Aut}(\overline{\mathbb{Q}} \mid \mathbb{Q}) \backslash\{\operatorname{id}\}$.

We now introduce the transcendental meromorphic function

$$
W(z):=\sum_{k=1}^{\infty} \frac{\beta^{k}}{z-\alpha_{t} q^{k}}
$$

with all poles in $|z|>1$. The two main properties of this function we will need are

$$
\frac{W\left(q^{-n}\right)}{q^{n}}=\beta^{-n}\left(\omega_{t}-\sum_{k=1}^{n} \frac{\beta^{k}}{1-\alpha_{t} q^{k}}\right) \quad\left(n \in \mathbb{N}_{0}\right)
$$

and

$$
\frac{W^{(\mu)}(0)}{\mu !}=\frac{\beta}{\alpha_{t}^{\mu+1}\left(\beta-q^{\mu+1}\right)} \quad\left(\mu \in \mathbb{N}_{0}\right) .
$$

With a parameter $N \in \mathbb{N}$ to be fixed later large enough, we now consider the following integral

$$
J:=J(N):=\frac{1}{2 \pi i} \oint_{|z|=1} \frac{\prod_{\ell=1}^{N}\left(z-\alpha_{t} q^{\ell}\right)}{z^{N} \prod_{n=1}^{N}\left(1-q^{n} z\right)} W(z) d z,
$$

where the integration path is in the positive sense. Evidently, the poles of the integrand in $|z|<1$ are exactly at 0 and at $q^{-n}(n=1, \ldots, N)$; the poles in $|z| \geq 1$ lie exactly at $\alpha_{t} q^{k}(k=N+1, N+2, \ldots)$.

These latter poles outside the unit disk lead to a series representation and to an asymptotic evaluation of the integral (14). Namely, we have from (14) and the above definition of $W$

$$
J=-\sum_{k>N} \frac{\beta^{k} \prod_{\ell=1}^{N}\left(q^{k}-q^{\ell}\right)}{q^{N k} \prod_{n=1}^{N}\left(1-\alpha_{t} q^{n+k}\right)} .
$$


Notice that, by our hypothesis $\alpha \notin q^{-\mathbb{N}}$, all denominators in (15) are non-zero. Denoting the typical summand in (15) by $S_{k}$, we see for any $k>N$

$$
\frac{S_{k+1}}{S_{k}}=\frac{\beta\left(q^{k}-1\right)\left(1-\alpha_{t} q^{k+1}\right)}{q^{N}\left(q^{k-N}-1\right)\left(1-\alpha_{t} q^{k+N+1}\right)},
$$

hence $\left|S_{k+1} / S_{k}\right| \leq \delta_{1}|q|^{-N}$, where $\delta_{1}>0$ (and all $\delta_{i}$ later in this section) is independent of $N$ and $k$. This, (15) and

$$
\left|S_{N+1}\right|=\left|\frac{\beta}{\alpha_{t}}\right|^{N}|q|^{-\frac{3}{2} N(N+1)} \delta_{2}\left(1+\mathrm{O}\left(|q|^{-N}\right)\right)
$$

with O-constant (here and later in this section) independent of $N$ gives us, for $N$ large enough,

$$
\frac{1}{2} \delta_{2}\left|\frac{\beta}{\alpha_{t}}\right|^{N}|q|^{-\frac{3}{2} N(N+1)} \leq|J| \leq 2 \delta_{2}\left|\frac{\beta}{\alpha_{t}}\right|^{N}|q|^{-\frac{3}{2} N(N+1)} .
$$

Notice that, for our purely qualitative considerations, we will not use the full left-hand side inequality but only $J=J(N) \neq 0$ for any large $N$.

On the other hand, using the poles inside the unit disk, we obtain from (14)

$$
\begin{gathered}
J=\sum_{n=1}^{N} \frac{(-1)^{N-n+1}}{\beta^{n}} \cdot \frac{q^{\frac{1}{2} n(n-1)} \prod_{\ell=1}^{N}\left(1-\alpha_{t} q^{\ell+n}\right)}{\prod_{\nu=1}^{n-1}\left(q^{\nu}-1\right) \prod_{\nu=1}^{N-n}\left(q^{\nu}-1\right)}\left(\omega_{t}-\sum_{k=1}^{n} \frac{\beta^{k}}{1-\alpha_{t} q^{k}}\right) \\
+\left.\left.\sum_{\kappa+\lambda+\mu=N-1} \frac{1}{\kappa !}\left(\frac{d}{d z}\right)^{\kappa} \prod_{\ell=1}^{N}\left(z-q^{\ell}\right)\right|_{z=0} \cdot \frac{1}{\lambda !}\left(\frac{d}{d z}\right)^{\lambda} \prod_{n=1}^{N}\left(1-q^{n} z\right)^{-1}\right|_{z=0} \cdot \frac{\alpha_{t}^{\lambda}}{1-\beta^{-1} q^{\mu+1}} .
\end{gathered}
$$

Here we applied also (12) and (13). Notice that, in the last triple sum, the summation is over all $(\kappa, \lambda, \mu) \in \mathbb{N}_{0}^{3}$ with $\kappa+\lambda+\mu=N-1$. We will soon need some informations on the two derivatives at the origin occurring here in the triple sum, which we state next.

Lemma 1. All numbers

$$
c_{\kappa}:=\left.\frac{1}{\kappa !}\left(\frac{d}{d z}\right)^{\kappa} \prod_{\ell=1}^{N}\left(z-q^{\ell}\right)\right|_{z=0} \quad \text { and } \quad d_{\lambda}:=\left.\frac{1}{\lambda !}\left(\frac{d}{d z}\right)^{\lambda} \prod_{n=1}^{N}\left(1-q^{n} z\right)^{-1}\right|_{z=0}
$$

arising in $(17)$ are in $\mathbb{Z}[q]$, and the absolute values of their $\sigma$ th conjugate, $\sigma \in \operatorname{Aut}(\overline{\mathbb{Q}} \mid \mathbb{Q}) \backslash$ $\{\mathrm{id}\}$, are bounded above by a positive constant depending only on $\left|q^{\{\sigma\}}\right|$.

Proof of Lemma 1. We exemplify it only for $d_{\lambda}$ since, for $c_{\kappa}$, it is rather similar. In some neighborhood of the origin we have

$$
\prod_{n=1}^{N}\left(1-q^{n} z\right)^{-1}=\sum_{\lambda=0}^{\infty} d_{\lambda} z^{\lambda} \quad \text { with } \quad d_{\lambda}=\sum_{\lambda_{1}+\ldots+\lambda_{N}=\lambda} q^{1 \cdot \lambda_{1}+\ldots+N \cdot \lambda_{N}},
$$

whence, using $\left|q^{\{\sigma\}}\right|<1$,

$$
\left|d_{\lambda}^{\{\sigma\}}\right| \leq \sum_{\lambda=0}^{\infty}\left|d_{\lambda}^{\{\sigma\}}\right| \leq \sum_{\lambda=0}^{\infty} \sum_{\lambda_{1}+\ldots+\lambda_{N}=\lambda}\left|q^{\{\sigma\}}\right|^{1 \cdot \lambda_{1}+\ldots+N \cdot \lambda_{N}}=\prod_{n=1}^{N}\left(1-\left|q^{\{\sigma\}}\right|^{n}\right)^{-1} .
$$


Here the last product is bounded above by $\prod_{n=1}^{\infty}\left(1-\left|q^{\{\sigma\}}\right|^{n}\right)^{-1}$.

Returning to the proof of Theorem 1, we obtain from (17)

$$
J=\omega_{t} \Sigma_{1}+\Sigma_{2}+\Sigma_{3}
$$

where we put

$$
\Sigma_{1}:=\sum_{n=1}^{N} T_{n} \quad \text { with } \quad T_{n}:=\sum_{n=1}^{N} \frac{(-1)^{N-n+1}}{\beta^{n}} \cdot \frac{q^{\frac{1}{2} n(n-1)} \prod_{\ell=1}^{N}\left(1-\alpha_{t} q^{\ell+n}\right)}{\prod_{\nu=1}^{n-1}\left(q^{\nu}-1\right) \prod_{\nu=1}^{N-n}\left(q^{\nu}-1\right)}
$$

for $n=1, \ldots, N$, and furthermore

$$
\Sigma_{2}:=-\sum_{n=1}^{N} T_{n} \sum_{k=1}^{n} \frac{\beta^{k}}{1-\alpha_{t} q^{k}}, \quad \Sigma_{3}:=\sum_{\kappa+\lambda+\mu=N-1} \frac{c_{\kappa} \alpha_{t}^{\lambda} d_{\lambda}}{1-\beta^{-1} q^{\mu+1}} .
$$

Next, we are looking for a denominator for $\Sigma_{1}, \Sigma_{2}, \Sigma_{3}$. To that purpose, we introduce the number

$$
D:=D(N):=\prod_{\nu=1}^{N-1}\left(q^{\nu}-1\right) \cdot \prod_{k=1}^{N}\left(1-\alpha_{t} q^{k}\right) \cdot \prod_{\mu=1}^{N}\left(1-\beta^{-1} q^{\mu}\right),
$$

which is in $\mathbb{Z}\left[q, \alpha_{t}, \beta\right]$ since $\beta$ is a unit. The asymptotic evaluation of $D$ gives

$$
|D|=\left|\frac{\alpha_{t}}{\beta}\right|^{N}|q|^{\frac{3}{2} N^{2}+\frac{1}{2} N} \cdot \delta_{3} \cdot\left(1+\mathrm{O}\left(|q|^{-N}\right)\right),
$$

and combining this with (16) we obtain (much more than)

$$
0<|D \cdot J|<3 \delta_{2} \delta_{3}|q|^{-N}
$$

if $N$ is large enough. According to (18) and (19) we find

$$
D \cdot J=\omega_{t}\left(D \cdot \Sigma_{1}\right)+D \cdot\left(\Sigma_{2}+\Sigma_{3}\right)
$$

with all $D \cdot \Sigma_{j} \in \mathbb{Z}\left[q, \alpha_{t}, \beta\right]$, where the degrees in $\alpha_{t}$ are bounded above by $2 N$.

Assuming now $\omega_{t} \in K$ we get $D \cdot J \in K^{\times}$and, moreover,

$$
\Psi:=\operatorname{den}\left(\omega_{t}\right) \cdot\left(\operatorname{den}\left(\alpha_{t}\right)\right)^{2 N} \cdot(D \cdot J) \in O_{K} \backslash\{0\}
$$

from which we see, by (21),

$$
|\Psi| \leq \delta_{4}\left(\operatorname{den}\left(\alpha_{t}\right)\right)^{2 N}|q|^{-N}
$$

We finally have to bound the absolute values of the conjugates of $\Psi$, hence, by (22) and (18), those of $D, \Sigma_{1}, \Sigma_{2}, \Sigma_{3}$. In virtue of $\left|\beta^{\{\sigma\}}\right| \geq 1 \geq\left|\alpha_{t}^{\{\sigma\}}\right|$ and $\left|q^{\{\sigma\}}\right|<1$ for any $\sigma \neq$ id, we easily obtain $\left|D^{\{\sigma\}}\right|,\left|\Sigma_{1}^{\{\sigma\}}\right|,\left|\Sigma_{2}^{\{\sigma\}}\right| \leq \delta_{5}$ from (19) and the definition of $\Sigma_{1}, \Sigma_{2}$ after (18), respectively. Since $\Sigma_{3}$ has $\mathrm{O}\left(N^{2}\right)$ summands, which are uniformly bounded above by some constant of type $\delta$, we find

$$
\left|\Psi^{\{\sigma\}}\right| \leq \delta_{6} N^{2}\left(\operatorname{den}\left(\alpha_{t}\right)\right)^{2 N}
$$


for any $\sigma \neq$ id. Thus, by (22) and (23),

$$
1 \leq\left|\operatorname{Norm}_{K \mid \mathbb{Q}}(\Psi)\right| \leq \delta_{7} N^{2(\operatorname{deg}(q)-1)}\left(\operatorname{den}\left(\alpha_{t}\right)\right)^{2 N \operatorname{deg}(q)}|q|^{-N},
$$

and, since $|q|>\left(\operatorname{den}\left(\alpha_{t}\right)\right)^{2 \operatorname{deg}(q)}$, this is a contradiction for every large $N$, and Theorem 1 is proved.

Remark. Since $|J|=|q|^{-3 N^{2} / 2+O(N)}$ and $|D|=|q|^{3 N^{2} / 2+O(N)}$ according to (16) and (20), respectively, the final contradiction can be reached only by a sharp evaluation of these two O-constants. More precisely, the proof goes only through since $0 \neq|D \cdot J|=\mathrm{O}\left(|q|^{-\delta N}\right)$ can be saved with some $\delta>0$ (in fact, with $\delta=1$, compare (21)) leading to the denominator condition in Theorem 1. This is noteworthy since, in most other arithmetical proofs in the $q$-'business', the contradictions are obtained by comparing the constant factors of $N^{2}$ in the exponents of $|q|$ (see, e.g., [3],[4],[5],[12][13],[15]).

\section{Some analytic facts on the functions $\mathcal{R}_{\ell}(z ; a, b)$}

To get explicitly the meromorphic continuation of the function $\mathcal{R}(z ; a, b)$ originally defined in $|z|<|u|^{a}$ by the power series (4), we provide the following lemma.

Lemma 2. If $q \in \mathbb{C}^{\times}$is fixed, then for any $z, w \in \mathbb{C}$ with $|z|<|q|$ and $|w q|>1$ the following identity holds

$$
\sum_{n=1}^{\infty} \frac{z^{n}}{1-w q^{n}}=z \sum_{n=1}^{\infty} \frac{w^{-n}}{z-q^{n}}
$$

Remark. If $0<|z|, \frac{1}{|w|}<|q|$ this identity can be written symmetrically as

$$
\sum_{n=1}^{\infty} \frac{z^{n}}{1-w q^{n}}=\sum_{n=1}^{\infty} \frac{\left(\frac{1}{w}\right)^{n}}{1-\left(\frac{1}{z}\right) q^{n}}
$$

where one side results from the other by replacing $(z, w)$ by $\left(\frac{1}{w}, \frac{1}{z}\right)$.

Proof. Since $|w q|>1$ the left-hand side of (24) equals

$$
-\sum_{n=1}^{\infty} \frac{z^{n}}{w q^{n}\left(1-\frac{1}{w q^{n}}\right)}=-\sum_{n=1}^{\infty} z^{n} \sum_{j=1}^{\infty}\left(w q^{n}\right)^{-j}=-\sum_{j=1}^{\infty} w^{-j} \sum_{n=1}^{\infty}\left(z q^{-j}\right)^{n} .
$$

Closed summation of the last inner sum leads to the right-hand side of (24).

Lemma 3. The function $\mathcal{R}(z ; a, b)$ occurring in Theorem 2 and defined in $|z|<|u|^{a}$ by the power series (4) can be continued meromorphically over $\mathbb{C}$. The continuation has poles exactly at the points $u^{a}\left(\frac{u}{v}\right)^{a m}, m \in \mathbb{N}_{0}$, and these are all simple.

Proof. For $N \in \mathbb{N}_{0}$ we have

$$
\mathcal{R}(z ; a, b)-\sum_{n=1}^{N} \frac{z^{n}}{R_{a n+b}}=\frac{z^{N}}{h v^{a N+b}} \sum_{k=1}^{\infty} \frac{\left(v^{-a} z\right)^{k}}{1-\left(-\frac{g}{h}\left(\frac{u}{v}\right)^{a N+b}\right)\left(\frac{u}{v}\right)^{a k}}
$$


in $|z|<|u|^{a}$. To the series on the right-hand side over $k$ we apply the identity (24) after replacing $q \rightarrow\left(\frac{u}{v}\right)^{a}, z \rightarrow \frac{z}{v^{a}}, w \rightarrow-\frac{g}{h}\left(\frac{u}{v}\right)^{a N+b}$. To satisfy the condition $|w q|>1$ in Lemma 2 , we choose $N \in \mathbb{N}_{0}$ according to

$$
\left|\frac{g}{h}\left(\frac{u}{v}\right)^{a N+b}\right| \geq 1
$$

which is possible by $|u|>|v|$ and $a>0$. Then Lemma 2 and (25) together yield

$$
\mathcal{R}(z ; a, b)=\sum_{n=1}^{N} \frac{z^{n}}{R_{a n+b}}+\frac{z^{N+1}}{h v^{a N+b}} \sum_{k=1}^{\infty} \frac{\left(-\frac{h}{g}\left(\frac{v}{u}\right)^{a N+b}\right)^{k}}{z-v^{a}\left(\frac{u}{v}\right)^{a k}},
$$

whence our lemma.

Next, we consider the generalizations (1) of (4) defining in $|z|<|u|^{a \ell}$ the function $\mathcal{R}_{\ell}(z ; a, b)$. Plainly, $\mathcal{R}_{1}(z ; a, b)$ is our earlier $\mathcal{R}(z ; a, b)$. We now proceed to clarify the connection between $\mathcal{R}_{\ell+1}$ and $\mathcal{R}_{\ell}$. To this purpose we multiply the obvious identity

$$
R_{a(n+\ell)+b}-v^{a \ell} R_{a n+b}=g u^{a n+b}\left(u^{a \ell}-v^{a \ell}\right)
$$

by $z^{n} /\left(R_{a n+b} \cdot \ldots \cdot R_{a(n+\ell)+b}\right)$ and then sum over all $n \in \mathbb{N}$. As result we obtain

$$
\sum_{n=1}^{\infty} \frac{z^{n}}{\prod_{\nu=n}^{n+\ell-1} R_{a \nu+b}}-\frac{v^{a \ell}}{z} \sum_{n=1}^{\infty} \frac{z^{n+1}}{\prod_{\nu=n+1}^{n+\ell} R_{a \nu+b}}=g u^{b}\left(u^{a \ell}-v^{a \ell}\right) \sum_{n=1}^{\infty} \frac{\left(u^{a} z\right)^{n}}{\prod_{\nu=n}^{n+\ell} R_{a \nu+b}},
$$

which can be written as

$$
\mathcal{R}_{\ell}(z ; a, b)-\frac{v^{a \ell}}{z}\left(\mathcal{R}_{\ell}(z ; a, b)-\frac{z}{\prod_{\nu=1}^{\ell} R_{a \nu+b}}\right)=g u^{b}\left(u^{a \ell}-v^{a \ell}\right) \mathcal{R}_{\ell+1}\left(u^{a} z ; a, b\right)
$$

or equivalently as

$$
\left(1-\frac{v^{a \ell}}{z}\right) \mathcal{R}_{\ell}(z ; a, b)+\frac{v^{a \ell}}{\prod_{\nu=1}^{\ell} R_{a \nu+b}}=g u^{b}\left(u^{a \ell}-v^{a \ell}\right) \mathcal{R}_{\ell+1}\left(u^{a} z ; a, b\right) .
$$

From this identity and Lemma 3 it is inductively clear that every power series (1) can be continued meromorphically from $|z|<|u|^{a \ell}$ to the whole complex plane. The poles of the resulting function $\mathcal{R}_{\ell}(z ; a, b)$ are exactly at the points $u^{a \ell}\left(\frac{u}{v}\right)^{a m}, m \in \mathbb{N}_{0}$, and they are all simple. For $\ell=1$, this follows from Lemma 3. As soon as this is true for $\ell$, then $\mathcal{R}_{\ell}(z ; a, b)$ certainly has no pole at $z=v^{a \ell}$, and we learn from (28): $p \in \mathbb{C}^{\times}$is a pole of $\mathcal{R}_{\ell+1}(z ; a, b)$ if and only if $p u^{-a}$ is a pole of $\mathcal{R}_{\ell}(z ; a, b)$, hence exactly if $p u^{-a}=u^{a \ell}\left(\frac{u}{v}\right)^{a m}$ with suitable $m \in \mathbb{N}_{0}$. Thus we find $p \in u^{a(\ell+1)}\left(\frac{u}{v}\right)^{a \mathbb{N}_{0}}$ and also the simplicity of all these poles.

Next, we want to express $\mathcal{R}_{\ell}(z ; a, b)$, for every $\ell \in \mathbb{N}$, by the function $\mathcal{R}(z ; a, b)$.

Lemma 4. Defining

$$
A_{\lambda}:=\frac{v^{a \lambda}}{g u^{b}\left(u^{a \ell}-v^{a \ell}\right) \prod_{\nu=1}^{\lambda} R_{a \nu+b}} \quad \text { and } \quad B_{\lambda}(z):=\frac{z-v^{a \lambda}}{z g u^{b}\left(u^{a \lambda}-v^{a \lambda}\right)},
$$


the following identity holds for any $\ell \in \mathbb{N}$

$$
\mathcal{R}_{\ell}(z ; a, b)=\mathcal{R}\left(\frac{z}{u^{a(\ell-1)}} ; a, b\right) \prod_{\lambda=1}^{\ell-1} B_{\lambda}\left(\frac{z}{u^{a(\ell-\lambda)}}\right)+\sum_{\lambda=1}^{\ell-1} A_{\lambda} \prod_{\kappa=\lambda+1}^{\ell-1} B_{\kappa}\left(\frac{z}{u^{a(\ell-\kappa)}}\right) .
$$

Proof. (30) being trivial for $\ell=1$, assume now that it is valid for some $\ell \in \mathbb{N}$. By (29), formula (28) is equivalent to

$$
\mathcal{R}_{\ell+1}(z ; a, b)=B_{\ell}\left(\frac{z}{u^{a}}\right) \mathcal{R}_{\ell}\left(\frac{z}{u^{a}} ; a, b\right)+A_{\ell} .
$$

Substituting here on the right-hand side for $\mathcal{R}_{\ell}\left(z / u^{a} ; a, b\right)$ the suitably modified right-hand side of (30), we obtain (30) with $\ell+1$ instead of $\ell$.

Although our next lemma is purely arithmetical, we shall need it to prove Lemma 6 on the functions $\mathcal{F}_{\ell}(z ; 1,0)$ and $\mathcal{L}_{\ell}(z ; 1,0)$, the two meromorphic functions generated by the power series (1) in the particular cases $R_{m}=F_{m}$ and $R_{m}=L_{m}$, respectively.

Lemma 5. For all $k, n \in \mathbb{N}_{0}$ the following identities hold

$$
\begin{aligned}
F_{n+2 k} F_{n+2 k+1}+F_{n} F_{n+1}-L_{2 k} F_{n} F_{n+2 k+1} & =(-1)^{n} F_{2 k} F_{2 k+1}, \\
L_{n+2 k} L_{n+2 k+1}+L_{n} L_{n+1}-L_{2 k} L_{n} L_{n+2 k+1} & =-5(-1)^{n} F_{2 k} F_{2 k+1} .
\end{aligned}
$$

Proof. To begin with, we quote the following formulas, which are valid for all $m, j \in \mathbb{N}_{0}$ and can be easily verified by induction using the recurrence relation of the $F$ 's and $L$ 's, respectively.

$$
\begin{array}{cc}
F_{m+1}^{2}-F_{m} F_{m+1}-F_{m}^{2}=(-1)^{m}, & L_{m+1}^{2}-L_{m} L_{m+1}-L_{m}^{2}=-5(-1)^{m}, \\
F_{m+j}=F_{j-1} F_{m}+F_{j} F_{m+1}, & L_{m+j}=F_{j-1} L_{m}+F_{j} L_{m+1}
\end{array}
$$

with the convention $F_{-1}:=1$. Denoting now the left-hand side of (31) by $\Delta_{n, k}$, the first formula in (34) leads to

$$
\begin{gathered}
\Delta_{n, k}=\left(\left(F_{2 k-1}-L_{2 k}\right) F_{n}+F_{2 k} F_{n+1}\right)\left(F_{2 k} F_{n}+F_{2 k+1} F_{n+1}\right)+F_{n} F_{n+1} \\
=F_{2 k} F_{2 k+1}\left(F_{n+1}^{2}-F_{n}^{2}\right)+\left(F_{2 k}^{2}-F_{2 k+1}^{2}+1\right) F_{n} F_{n+1}=F_{2 k} F_{2 k+1}\left(F_{n+1}^{2}-F_{n} F_{n+1}-F_{n}^{2}\right),
\end{gathered}
$$

where we used $F_{m-1}+F_{m+1}=L_{m}(m \geq 0)$ and the first equation in (33) with $m=2 k$. Again by the first equation (33), the expression in the last brackets equals $(-1)^{m}$, whence identity (31).

The proof of (32) is similar. Of course, instead of the first formula in (34) we have to use the second one, and later one needs both equations (33), each once.

The analytic conterpart of Lemma 5 reads as follows.

Lemma 6. For each $k \in \mathbb{N}$, the functions $\mathcal{F}_{2 k}$ and $\mathcal{L}_{2 k}$ satisfy the following recursion formulas:

$$
F_{2 k} F_{2 k+1} z^{2} \mathcal{F}_{2 k+2}(-z ; 1,0)=\left(z^{2}+1-L_{2 k} z\right) \mathcal{F}_{2 k}(z ; 1,0)+\frac{z\left(F_{4 k+1} z-F_{2 k+1}\right)}{F_{1} \cdot \ldots \cdot F_{2 k+1}},
$$




$$
-5 F_{2 k} F_{2 k+1} z^{2} \mathcal{L}_{2 k+2}(-z ; 1,0)=\left(z^{2}+1-L_{2 k} z\right) \mathcal{L}_{2 k}(z ; 1,0)+\frac{z\left(L_{4 k+1} z-L_{2 k+1}\right)}{L_{1} \cdot \ldots \cdot L_{2 k+1}} .
$$

Proof. After multiplication of equation (31) by $z^{n} /\left(F_{n} \cdot \ldots \cdot F_{n+2 k+1}\right)$, we sum over all $n \in \mathbb{N}$ to get (35). To obtain the term $F_{4 k+1}$ on the right-hand side we used $L_{m} F_{m+1}=$ $F_{2 m+1}+(-1)^{m}\left(m \in \mathbb{N}_{0}\right)$ for $m=2 k$. Plainly, to demonstrate (36) we multiply (32) by $z^{n} /\left(L_{n} \cdot \ldots \cdot L_{n+2 k+1}\right)$, proceed then as before but use $L_{m} L_{m+1}=L_{2 m+1}+(-1)^{m}\left(m \in \mathbb{N}_{0}\right)$ for $m=2 k$ to get the term $L_{4 k+1}$ in (36).

Remark. Note that the two cases $z= \pm 1$ of (35) imply formulas of type $T_{\ell}=\rho_{1}+\rho_{2} S_{\ell+2}$ and $S_{\ell}=\rho_{3}+\rho_{4} T_{\ell+2}$ with explicit $\rho_{1}, \rho_{2}, \rho_{3}, \rho_{4} \in \mathbb{Q}$, depending on $\ell$, and $\rho_{2} \rho_{4} \neq 0$, hence the full result of Melham [14]. Here $S_{\ell}$ and $T_{\ell}$ denote the sums in (9) and (10), respectively.

\section{Proof of Theorems 2, 3 and the corollaries}

\section{Proof of Theorem 2.}

Under the hypotheses of this theorem, the assertion (5) is equivalent, by (27), to

$$
\sum_{k=1}^{\infty} \frac{\left(-\frac{h}{g}\left(\frac{v}{u}\right)^{a N+b}\right)^{k}}{1-\frac{v^{a}}{\gamma}\left(\frac{u}{v}\right)^{a k}} \notin K,
$$

where $N \in \mathbb{N}_{0}$ has been chosen according to (26). Hence we may apply Theorem 1 to the sum (37) putting

$$
q:=\left(\frac{u}{v}\right)^{a}, \alpha:=\frac{v^{a}}{\gamma}, \beta:=-\frac{h}{g}\left(\frac{v}{u}\right)^{a N+b} .
$$

Of course, some enlargement of $N$ compared with (26) could be necessary to ensure also the hypothesis $\left|\beta^{\{\sigma\}}\right| \geq 1$ for any $\sigma \in \operatorname{Aut}(\overline{\mathbb{Q}} \mid \mathbb{Q}) \backslash\{\operatorname{id}\}$ in Theorem 1. The assumption $\left|\left(\frac{u}{v}\right)^{\{\sigma\}}\right|<1$ for all these $\sigma$ in Theorem 2 allows such a choice.

Proof of Theorem 3.

(i): If $\gamma$ satisfies all conditions of this case, then, by $(30)$, the assertion $\mathcal{R}_{\ell}(\gamma ; a, b) \notin K$ is equivalent to

$$
\mathcal{R}\left(\frac{\gamma}{u^{a(\ell-1)}} ; a, b\right) \notin K .
$$

This latter assertion holds, by (5) in Theorem 2, since

$$
\frac{\gamma}{u^{a(\ell-1)}} \notin u^{a}\left(\frac{u}{v}\right)^{a \mathbb{N}_{0}} \Leftrightarrow \gamma \notin u^{a \ell}\left(\frac{u}{v}\right)^{a \mathbb{N}_{0}}
$$

(the latter being satisfied by one of our hypotheses on $\gamma$ in case (i)) and the denominator condition in (i) is just the corresponding denominator condition at the end of Theorem 2 on $\gamma / u^{a(\ell-1)}$ instead of $\gamma$.

(ii): If $\gamma=u^{a\left(\ell-\lambda_{0}\right)} v^{a \lambda_{0}}$ for some $\lambda_{0} \in\{1, \ldots, \ell-1\}$, then we have for this $\lambda_{0}$, by (29), $B_{\lambda_{0}}\left(\gamma / u^{a\left(\ell-\lambda_{0}\right)}\right)=0$ and hence

$$
\mathcal{R}_{\ell}(\gamma ; a, b)=\sum_{\lambda=\lambda_{0}}^{\ell-1} A_{\lambda} \prod_{\kappa=\lambda+1}^{\ell-1} B_{\kappa}\left(u^{a\left(\kappa-\lambda_{0}\right)} v^{a \lambda_{0}}\right) \in K,
$$


by formula (30).

Proof of Corollary 1.

Denoting by $\varphi_{u} \in \mathbb{Z}[X]$ the monic minimal polynomial of $u$, our condition $\varphi_{u}(0) \in\{1,-1\}$ implies that $u$ is a unit in $O_{K}$, and we apply Theorem 3 with $v=1$. Therefore, we have only to verify the inclusion $K \subset \mathbb{Q}\left(u^{m}\right)$ for every $m \in \mathbb{N}$. Clearly, $\tilde{u}:=u^{m}$ is algebraic over $\mathbb{Q}$ of a degree dividing $\operatorname{deg}(u)=[K: \mathbb{Q}]$. It is well-known (and can be found in [10, p.69]) that the distinct conjugates of $\tilde{u}$ are the distinct numbers among the $\left(u^{\{\sigma\}}\right)^{m}, \sigma \in \operatorname{Aut}(\overline{\mathbb{Q}} \mid \mathbb{Q})$, and that here each conjugate of $\tilde{u}$ appears equally often. But this can occur only once since exactly one $\left|u^{\{\sigma\}}\right|$ is greater than 1 . Hence we have $\operatorname{deg}\left(u^{m}\right)=[K: \mathbb{Q}]$ for every $m \in \mathbb{N}$.

Proof of Corollary 2.

Since $\frac{u}{v}$ is either $\frac{A+\sqrt{\Delta}}{A-\sqrt{\Delta}}$ or its reciprocal we find $\left(\frac{u}{v}\right)^{m} \in \mathbb{Q}(\sqrt{\Delta}) \backslash \mathbb{Q}$ for any $m \in \mathbb{N}$, and hence all hypotheses of Theorem 3 are verified.

Proof of Corollary 3.

In the Fibonacci and Lucas cases, we have $u=\frac{1}{2}(1+\sqrt{5})\left(=\vartheta_{\infty}\right)$ and $v=\frac{1}{2}(1-\sqrt{5})$, whereas $(g, h)$ from $(2)$ is $\left(\frac{1}{\sqrt{5}},-\frac{1}{\sqrt{5}}\right)$ and $(1,1)$, respectively. Half of this we mentioned already at the beginning of section 1 . We add here only that the denominator of $\gamma^{-1} \vartheta_{\infty}^{a(2 t-1)}$ is 1 for any $\gamma= \pm \vartheta_{\infty}^{c}$.

Proof of Corollary 4.

In the notation introduced before Lemma 6 , the series $(9)$ and $(10)$ are $\mathcal{F}_{\ell}(1 ; 1,0)$ and $\mathcal{F}_{\ell}(-1 ; 1,0)$, respectively, whereas their Lucas analogues are $\mathcal{L}_{\ell}( \pm 1 ; 1,0)$. Because

$$
u^{\ell-\lambda} v^{\lambda}=(-1)^{\lambda} u^{\ell-2 \lambda}
$$

$\neq \pm 1$ for any $\lambda$, if $\ell$ is odd, the case (ii) of Corollary 2 can never occur; since $\pm u^{s}$ has denominator 1 for any $s \in \mathbb{Z}$, (i) of Corollary 2 gives the full information.

Hence, from now on, we may suppose $\ell$ even. Since (38) equals 1 exactly if $\lambda=\frac{\ell}{2}$ is even, respectively -1 exactly if $\lambda=\frac{\ell}{2}$ is odd, case (i) of Corollary 2 provides us the complete assertion if $4 \nmid \ell$ or $4 \mid \ell$, respectively. On the other hand, we know from case (ii) that

$$
\mathcal{F}_{\ell}(1 ; 1,0) \in \mathbb{Q}(\sqrt{5}) \text { if } 4 \mid \ell, \quad \text { and } \quad \mathcal{F}_{\ell}(-1 ; 1,0) \in \mathbb{Q}(\sqrt{5}) \text { if } 2 \mid \ell \text { but } 4 \not \ell,
$$

and the same for $\mathcal{L}_{\ell}$ instead of $\mathcal{F}_{\ell}$. In all situations, where case (ii) applies, we should be able to specify $\mathcal{F}_{\ell}( \pm 1 ; 1,0), \mathcal{L}_{\ell}( \pm 1 ; 1,0)$ entirely, and hence to see also that all $\mathcal{F}_{\ell}$-values in (39) are irrational (and their Lucas analogues as well). An appropriate tool for this, at least at first look, seems to be Lemma 4 . But the necessary evaluation of the rational functions $B_{\lambda}$ from (29) becomes rather unpleasant, hence we take a different way, also interesting by itself.

First, we compute $\mathcal{F}_{2}(-1 ; 1,0)$ via Lemma 4 . Since $B_{1}\left(-\frac{1}{u}\right)=B_{1}(v)=0$, by $(29)$, formula (30) leads to $\mathcal{F}_{2}(-1 ; 1,0)=A_{1}=v=\frac{1}{2}(1-\sqrt{5}) \in \mathbb{Q}(\sqrt{5}) \backslash \mathbb{Q}$. Applying now (35) from Lemma 6 with $k=1, z=-1$, we obtain

$$
F_{2} F_{3} \mathcal{F}_{4}(1 ; 1,0)=\left(2+L_{2}\right) \mathcal{F}_{2}(-1 ; 1,0)+\frac{F_{5}+F_{3}}{F_{1} F_{2} F_{3}},
$$


whence $\mathcal{F}_{4}(1 ; 1,0) \in \mathbb{Q}(\sqrt{5}) \backslash \mathbb{Q}$. Next, we apply (35) with $k=2, z=1$ to get

$$
F_{4} F_{5} \mathcal{F}_{6}(-1 ; 1,0)=\left(2-L_{4}\right) \mathcal{F}_{4}(1 ; 1,0)+\frac{F_{9}-F_{5}}{F_{1} \cdot \ldots \cdot F_{5}},
$$

and the inductive procedure is clear.

In the Lucas case, we have the same $u, v$ as in the Fibonacci case but $g=1$ (instead of $\left.g=\frac{1}{\sqrt{5}}\right)$, hence again $B_{1}\left(-\frac{1}{u}\right)=0$ but $\mathcal{L}_{2}(-1 ; 1,0)=A_{1}=\frac{v}{(u-v) L_{1}}=\frac{1-\sqrt{5}}{2 \sqrt{5}}$. From here on, we conclude as above using (36) instead of (35).

\section{REFERENCES}

[1] R. André-Jeannin, Irrationalité de la somme des inverses de certaines suites récurrentes, C. R. Acad. Sci. Paris, Sér. I 308, 539-541 (1989).

[2] M. J. Bertin, A. Descomps-Guilloux, M. Grandet-Hugot, M. Pathiaux-Delefosse and J. P. Schreiber, Pisot and Salem numbers, Basel et al.: Birkhäuser, 1992.

[3] P. B. Borwein, On the irrationality of certain series, Math. Proc. Camb. Phil. Soc. 112, 141-146 (1992).

[4] P. Bundschuh, Linear independence of values of a certain Lambert series, Result. Math. 51, 29-42 (2007).

[5] P. Bundschuh and K. Väänänen, Arithmetical investigations of a certain infinite product, Compositio Math. 91, 175-199 (1994).

[6] L. Carlitz, Reduction formulas for Fibonacci summations, Fibonacci Q. 9, 449-466, 510-511 (1971).

[7] K. A. Driver and D. S. Lubinsky, Convergence of Padé approximants for a q-hypergeometric series (Wynn's power seriies III), Aequationes Math. 45, 1-23 (1993).

[8] D. Duverney, Ke. Nishioka, Ku. Nishioka and I. Shiokawa, Transcendence of Rogers-Ramanujan continued fraction and reciprocal sums of Fibonacci numbers, Proc. Japan Acad. Ser. A Math. Sci. 73, 140-142 (1997).

[9] C. Elsner, S. Shimomura and I. Shiokawa, Algebraic relations for reciprocal sums of Fibonacci numbers, Acta Arith. 130, 37-60 (2007).

[10] E. Hecke, Vorlesungen über die Theorie der algebraischen Zahlen, Leipzig: Akad. Verlagsgesellschaft, 1923; Engl. transl.: Lectures on the Theory of Algebraic Numbers, New York et al.: Springer, 1981.

[11] T. Matala-aho, Remarks on the arithmetic properties of certain hypergeometric series of Gauss and Heine, Acta Univ. Oulu. Ser. A Sci. Rerum Natur. 219, 1-112 (1991).

[12] T. Matala-aho and M. Prévost, Irrationality measures for the series of reciprocals from recurrence sequences, J. Number Theory 96, 275-292 (2002).

[13] T. Matala-aho and M. Prévost, Quantitative irrationality for sums of reciprocals of Fibonacci and Lucas numbers, Ramanujan J. 11, 249-261 (2006).

[14] R. S. Melham, On some reciprocal sums of Brousseau: an alternative approach to that of Carlitz, Fibonacci Quart. 41, 59-62 (2003).

[15] M. Prévost, On the irrationality of $\sum t^{n} /\left(A \alpha^{n}+B \beta^{n}\right)$, J. Number Theory 73, 139-161 (1998).

[16] P. Ribenboim, My numbers, my friends, New York et al.: Springer, 2000.

[17] R. Salem, Algebraic Numbers and Fourier Analysis, Boston: Heath, 1963.

[18] Y. Tachiya, Irrationality of certain Lambert series, Tokyo J. Math. 27, 75-85 (2004).

[19] P. Wynn, A general system of orthogonal polynomials, Quart. J. Math. Oxford Ser. 18, 81-96 (1967).

Rauchfreie Universität Köln, Mathematisches Institut, Weyertal 86-90, 50931 Köln, Germany E-mail address: pb@math.uni-koeln.de

Received on 22-07-08

Accepted on 01-08-08 